\title{
Output Power of Linear Generators under Reactive Control in Regular Waves
}

\author{
Agustín García Santana ${ }^{1}$, Dan El Montoya Andrade ${ }^{2}$ and Antonio de la Villa Jaén ${ }^{3}$ \\ ${ }^{1}$ Department of Electrical Engineering \\ AG Ingeniería \\ Phone:+0034 634 821844, e-mail: agustin.garcia@agingenieria.abengoa.com
}

${ }^{2}$ Department of Electrical Engineering

Venezuela Central University

e-mail: danel.montoya@ucv.ve

${ }^{3}$ Department of Electrical Engineering

E.S.I., Seville University

e-mail: adelavilla@us.es

\begin{abstract}
Direct drive wave energy converters couple a linear generator directly to a reciprocating wave energy device. This way, the performances showed by hydraulic and pneumatic based systems are improved. After linear generator, full-scale back-to-back Voltage Source Converters are used to adequate the electric energy before delivering to the grid. This stage is made up of two parts: the generator side converter stage consists of rectifying the electricity generated by the linear generator; the other one turns it in alternating current with suitable voltage and frequency parameters. The first one allows control over the instantaneous power flow possible, and in turn enables both power flow directions; this capability allows implementing reactive control. This control strategy is used to maximize the energy extraction from waves by the power takeoff (PTO) based on reaction force control. This paper shows how to determine and quantify, not the power extracted from waves, but the one transferred to the electronic converter.
\end{abstract}

\section{Key words}

Wave energy converters, linear generators, WEC control, renewable energy.

\section{Introduction}

Ocean energy conversion systems have seen renewed interest, stimulated mostly by the increasing energy discussions. Several commercial ocean wave energy projects have already been undertaken. Sea waves are a very promising energy carrier among renewable power sources, since they are able to manifest an enormous amount of energy resources in almost all geographical regions.

A diversity of prototypes has been developed during the last decades. This paper focuses on oscillating buoy systems, which are a kind of oscillating point absorber [1]. In these systems, waves exert forces on these devices, and the vertical axis movement can be exploited.
The oscillating system consists of a buoy on the ocean surface connected to a Permanent Magnet Linear Generator (PMLG) with a rope set on the seabed. Permanent magnets are mounted on the traslator and the varying magnetic field acts on stator windings. Here the generator can have different number of sides and the stator ones are fixed to the foundation on the seabed. Springs are connected to the alternator. The generator is placed in a watertight enclosure.

Device survival possibilities are increased because of the direct drive PTO system. It highlights simplicity and robustness, and also reduces the maintenance costs and probably those incurred because of extracting energy from waves. The available primary power features are pulsing and thus, the electric energy should be set up to be properly injected into the grid. Linear generators and full-scale back-to-back Voltage Source Converters (VSC), implemented together, are one of the most promising choices used to connect the electricity generated by buoy oscillating systems to the power grid. The buoy oscillating motion can be directly transformed into electricity applying linear generators, without conversion to rotative motion. This way, mechanical linkages are avoided and efficiency increases. DC link capacitor can also be used as an intermediate stage of storage through electronics power, and thus, electric power can be tuned to grid connection parameters. Figure 1 shows the general scheme.

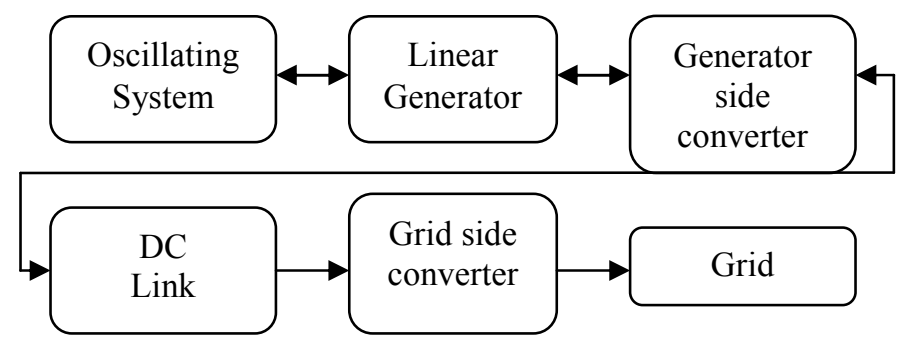

Fig. 1: General WEC system scheme. 
The amount of energy extractable from waves depends on the hydrodynamic characteristics of the WEC system, which consist of buoy and generator moving parts. Taking into account the type of WEC and the incident wave, optimal hydrodynamic characteristics can be found in order to achieve the maximum amount of energy when Linear Waves Theory is applied.

\section{Hydrodynamic control}

In linear wave theory, the equation which models the oscillating system motion with a single degree of freedom in regular waves moving in heave is [2]:

$$
f_{e}+f_{p t o}=\left(m+m_{\text {add }}\right) \ddot{z}+b \dot{z}+\left(\rho g S+k_{s}\right) z
$$

where $z$ is the vertical displacement of the oscillating system, $f_{e}$ is the excitation force resulting from the incident wave, and the force applied on the buoy by the PTO mechanism is $f_{p t o}$, which can be expressed as a function of parameters $m_{p t o}, r_{p t o}$ and $k_{p t o}$, as follows:

$$
f_{p t o}=-m_{p t o} \ddot{z}-r_{p t o} \dot{z}-k_{p t o} z
$$

This approximation is only possible if the kind of control applied to the PTO is linear. Taking (2) account in (1), we have:

$$
f_{e}=\left(m+m_{\text {add }}+m_{p t o}\right) \ddot{z}+\left(r_{p t o}+b\right) \dot{z}+\left(\rho g S+k_{s}+k_{p t o}\right) z
$$

Values of parameters $m_{\text {pto }}, r_{\text {pto }}$ and $k_{\text {pto }}$ depend on the control strategy chosen for the PTO. Damping effect is related to $r_{p t o}$ parameter whereas $m_{\text {pto }}$ and $k_{p t o}$ parameters are related to inertial and elastic effects respectively.

Further, $m$ is the mass of the floater including all moving parts of the generator, $\rho$ is the water density, and $g$ the acceleration of gravity. The hydrodynamics parameter $m_{\text {add }}$ and $b$ are the added mass representing the water above the floater that has to be accelerated, and the radiation damping coefficient, which includes radiation of waves by the oscillating body. These parameters determine the hydrodynamic characteristics of the buoy and depend on the frequency of the incident wave. Moreover $S$ is the water plane area of the buoy and $k_{s}$ the spring constant that ties the device to the seabed.

In regular waves of angular frequency $\omega$ and using linear PTO, it is convenient to apply complex representation involving complex amplitudes and phasors. In this paper, the following phasor representation is used:

$$
\hat{X}=X e^{j \theta}
$$

where $j$ is the imaginary unit and $X$ is the root-meansquare dimension value.

The dynamic of a WEC point absorber can be represented using electrical analogue of the mass-springdamper system to analyze power intercepted and captured [3]. Fig. 2 shows a series circuit representing the
WEC where the electrical equivalent of the wave excitation force, $f_{e}$ is represented by a EMF source. This EMF source generates a current analogous with the device velocity $\dot{z}$, whose rms value has been called $U$ in Fig. 2.

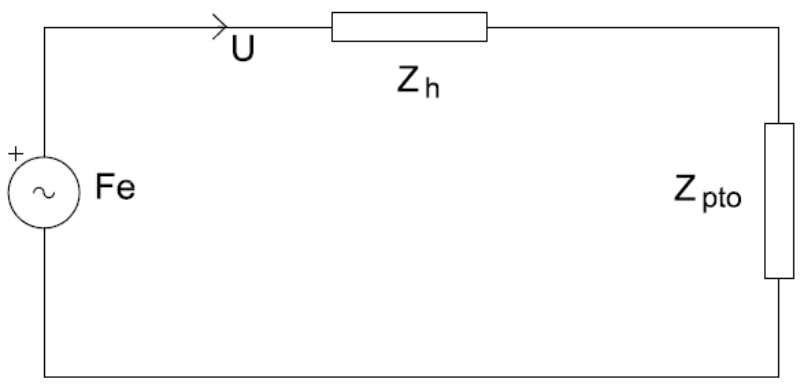

Fig. 2: Electrical analogue of a point absorber.

The impedances shown in the circuit are calculated by:

$$
\begin{gathered}
Z_{h}=b+j\left[\omega\left(m+m_{a d d}\right)-\frac{\rho g S+k_{s}}{\omega}\right] \\
Z_{p t o}=r_{p t o}+j\left[\omega m_{p t o}-\frac{k_{p t o}}{\omega}\right]
\end{gathered}
$$

Next, the parameters definitions are given:

- Inductance represents the sum of mass $m$ and added mass of the device $m_{\text {add }}$.

- Capacitance represents the inverse of the spring stiffness force constant $k_{s}$ and buoyancy $\rho g S$.

- Resistance represents the radiation damping $b$.

- Controllable and variable impedance $Z_{p t o}$ represents the reaction exerted by the PTO.

- The hydrodynamic impedance $Z_{h}$ represents the oscillating system.

The load impedance $Z_{p t o}$ can be expanded in terms of resistive, inductive, and capacitive equivalent components

Equation (1) can be expressed in frequency domain, as a function of the complex velocity, $\hat{U}=j \omega \hat{Z}$ as follows:

$$
\hat{F}_{e}+\hat{F}_{p t o}=Z_{h} \hat{U}
$$

where the intrinsic impedance is denoted by [2],

$$
Z_{h}=b+j x_{h}
$$

and

$$
x_{h}=\omega\left(m+m_{a d d}\right)-\frac{\rho g S+k_{s}}{\omega}
$$

In a similar way, (2) can be expressed by

$$
\hat{F}_{p t o}=-Z_{p t o} \hat{U}
$$


where the PTO complex impedance is used

$$
Z_{p t o}=r_{p t o}+j x_{p t o}
$$

and

$$
x_{p t o}=\omega m_{p t o}-\frac{k_{p t o}}{\omega}
$$

The net impedance of the oscillating system can be obtained as follows:

$$
Z_{n e t}=Z_{h}+Z_{p t o}=b+r_{p t o}+j\left(x_{h}+x_{p t o}\right)
$$

This way, excitation force and oscillating device velocity are related by

$$
\hat{F}_{e}=Z_{n e t} \hat{U}
$$

In regular waves, the excitation force for bodies with a vertical axis of symmetry, which are oscillating in heave on deep water, is given by [2]:

$$
F_{e}=\sqrt{\frac{2 g^{3} \rho b}{\omega^{3}}} \frac{H}{2}
$$

where $H$ is the incident wave height.

\section{Linear generator}

The $d q$ components [4] regarding to the linear generator model used in this paper can be expressed as follows:

$$
\begin{aligned}
& v_{d}=R_{s} i_{d}+L_{s} \frac{d i_{d}}{d t}-\omega_{m} L_{s} i_{q} \\
& v_{q}=R_{s} i_{q}+L_{s} \frac{d i_{q}}{d t}+\omega_{m} L_{s} i_{d}+\omega_{m} \psi
\end{aligned}
$$

where $R_{S}$ is the stator resistance, $L_{S}$ is the stator inductance and $\omega_{m}$ is the angular speed of the stator variables:

$$
\omega_{m}=\frac{2 \cdot \pi \cdot \dot{z}}{\lambda}
$$

where $\lambda$ is the pole width of the LPMG. Further $\psi$ is the flux linkage of the stator winding due to the flux produced by the permanent magnets. $v_{d}, v_{q}, i_{q}$ and $i_{d}$ are the voltage induced and the stator current, in the $d q$ reference frame.

The three-phase linear permanent magnet generator voltages output varies in both amplitude and frequency parameters [5]. Thus, it is necessary to use power electronics at the output generator, in order to modify these two parameters and, this way, to reach enough wave quality to deliver the electric power into the grid.

Also, there will be applied a control strategy to reduce losses in the generator equaling to zero the $i_{d}$ component.

$$
i_{d}=0
$$

Thus, the active power at the generator input [6] may be written as:

$$
p_{p t o}=\frac{3}{2} \cdot \omega_{m} \cdot \psi \cdot i_{q}
$$

The force applied on the buoy by the generator, may be calculated by the following expression [7]:

$$
f_{p t o}=\frac{p_{p t o}}{\dot{z}}=\frac{3 \cdot \pi \cdot \psi \cdot i_{q}}{\lambda}=\frac{3 \cdot \pi \cdot \psi \cdot i_{q}}{2 \tau_{p}}
$$

where $\tau_{p}$ is the pole pitch of the PMLG.

Note that the force developed by the PTO can be directly controlled by the quadrature component of the current $i_{q}$.

Taking into account (2), we have:

$$
i_{q}^{r e f}=-\frac{2 \tau_{p}}{3 \pi \psi}\left(m_{p t o} \ddot{z}+r_{p t o} \dot{z}+k_{p t o} z\right)
$$

\section{Reactive Control}

To further realise the benefits of a direct drive system, a control scheme based on reaction force control to maximise energy extraction can be used [2]. The force generated by a directly coupled linear electrical generator, the so-called generator reaction force, can be represented as three components: an inertial force proportional to the acceleration, a damping force proportional to velocity and a spring stiffness force proportional to displacement. Mechanical resonance occurs in a point absorber when the sum of the imaginary components of the net impedance adds up to zero. Maximum power transferred to the PTO occurs when the generator impedance is equal to the complex conjugate of the device impedance. Hence, the PTO impedances have to be controlled to meet the following requirements [3]:

$$
\begin{aligned}
& r_{p t o}=b \\
& x_{p t o}=-x_{h}
\end{aligned}
$$

where $x_{h}$ is calculated by (7).

\section{Proposed Formulation}

The average power extracted by the PTO device over a wave period, is given by 


$$
P_{p t o}=r_{p t o} U^{2}
$$

The proposed formulation aims to calculate the power transferred to the electronic converter. Thus, generator copper losses are included in it. In this section, this power is formulated as a function of the PTO control parameters. The power transferred from the linear generator to the electronic converter can be expressed by

$$
P_{c}=P_{p t o}-P_{\text {loss }}
$$

where $P_{\text {loss }}$ are the linear generator copper losses, which can be expressed as follows:

$$
P_{\text {loss }}=3 R_{s} I^{2}
$$

where $I$ is the current supplied by the linear generator to the electronic converter.

The current from the generator and the incident regular waves have the same period. The current can be determined from its $d q$ components [8], and considering (16) yields

$$
I^{2}=\frac{I_{q}^{2}}{2}
$$

Taking into account the expressions (18), (23), and (24), generator copper losses can be expressed by

$$
P_{\text {loss }}=\delta F_{p t o}^{2}
$$

where

$$
\delta=\frac{2 \tau_{p}^{2}}{3 \pi^{2} \psi^{2}} R_{s}
$$

Analyzing the circuit in Fig. 2, $F_{p t o}$ can be expressed as follows:

$$
F_{p t o}=\left|Z_{p t o}\right| U=\left|Z_{p t o}\right| \frac{F_{e}}{\left|Z_{n e t}\right|}
$$

using this last expression in (25), yields

$$
P_{l o s s}=\delta \cdot\left|Z_{p t o}\right|^{2} \frac{F_{e}^{2}}{\left|Z_{n e t}\right|^{2}}
$$

Thus, from expressions (21), (22) and (28), the power transferred from the linear generator to the electronic converter is

$$
P_{c}=\left(r_{p t o}-\delta \cdot\left|Z_{p t o}\right|^{2}\right) \frac{F_{e}^{2}}{\left|Z_{n e t}\right|^{2}}
$$

Note that if $R_{s}=0$, generator copper losses are neglected, and expressions (21) and (29) match up.

\section{Results}

In this section, the formulation developed before is applied to an oscillating system which size varies, when reactive control is used. It will quantify the energy captured by the PTO and the one which is transferred to the first converter. The difference between them lies on the power losses that occur in linear generator. The examples presented are based on hydrodynamic coefficients taken from reference [9]. These coefficients correspond to a variable radius heaving spherical buoy, which has a hemispherical wetted surface when there is no motion and no waves. The natural frequency, the added mass and the damping coefficient will depend on the radius value, which initially is 2.5 meters. The spring constant value is $6200 \mathrm{~N} / \mathrm{m}$.

The linear generator model implemented corresponds to the Lysekil Project, which has been developed at the Swedish Center for Renewable Electric Energy Conversion of Uppsala University [10]. Its main parameters are presented in table I.

Table I. - Main generator features

\begin{tabular}{|c|c|}
\hline Nominal output power & $10 \mathrm{~kW}$ \\
\hline Nominal speed & $0.7 \mathrm{~m} / \mathrm{s}$ \\
\hline Phase-to-phase voltage r.m.s. & $200 \mathrm{~V}$ \\
\hline Pole width & $50 \mathrm{~mm}$ \\
\hline Synchronous reactance & $7.8 \mathrm{mH}$ \\
\hline Stator winding resistance & $0.45 \mathrm{ohms}$ \\
\hline
\end{tabular}

Results are obtained for regular waves, in this case the transported wave power per unit with of the wave front of period $\mathrm{T}$ and height $\mathrm{H}$ is [2]:

$$
J=\frac{\rho g^{2}}{32 \pi} T H^{2}
$$

The available incident wave power has been considered constant during the testing in order to compare the results. A reference value is assigned to the wave energy transport $J$. The incident wave height and the period are adopted according to (30). This way, the available power per meter of wave front matches up in every test. The reference value considered is $\mathrm{J}=5 \mathrm{~kW} / \mathrm{m}$. The incident wave period varies between $2.3 \mathrm{~s}$ and $3.6 \mathrm{~s}$. The resulting wave height, based on these assumptions, varies between $1.51 \mathrm{~m}$ and $1.20 \mathrm{~m}$.

\section{A. Power transferred to the PTO and to the electronic converter}

The power transferred to the PTO can be obtained from (23), whereas the power transferred to the electronic converter can be obtained from (29). Fig. 4 shows the average power extracted from the oscillating system and delivered to the PTO. It also depicts the average power transferred to the power electronic converter. 


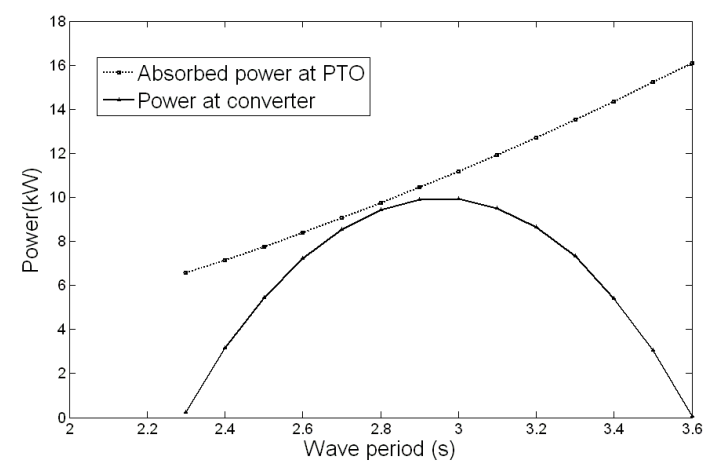

Fig. 4: Power absorbed at PTO and delivered to the first converter, versus wave period.

Note that, in the period bandwidth shown, the power absorbed at PTO increases with the wave period, whereas the power delivered to the first converter reaches a maximum point, and then diminishes when the wave period increases. The difference is due to the copper resistance that occurs in the linear generator.

In the case depicted, at $\mathrm{T}=3.6 \mathrm{~s}$, there is no average power flux through the power converter. Besides, the PTO receives $16 \mathrm{~kW}$. It means that the power absorbed from the PTO is not sent to the grid, when reactive control is applied. The reactive control strategy can be highly inefficient, and could be improved.

To match the resonance conditions required by the reactive control, may be extreme situations where the oscillating system and the PTO exchange large amounts of energy. When these exchanges are increasing, so are losses.

Note that, at $\mathrm{T}=2.79 \mathrm{~s}$. (natural frequency), both powers are very similar. It means that, at that frequency are hardly necessary reactive power exchanges and the energy flow goes permanently from the oscillating system to the power converter.

The power losses can be obtained from (28). Fig. 5 shows the losses associated with the linear generator. Note that when the losses in linear generator increase, the power transferred to the converter diminishes and vice versa. Note that the power that can be transferred to the electronic converter can be null, or even changes its sign when losses notably increase $(\mathrm{T}=3.6 \mathrm{~s}$. in figure 5$)$.

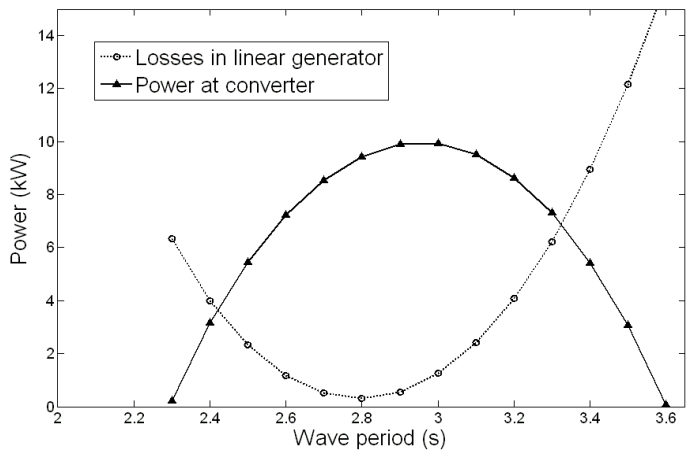

Figure 5: Power losses at linear generator

\section{B. System response versus different values of the sphere radius}

Table II shows different results when the sphere radius is modified, holding fixed the generator resistance.

Table II. - Power and periods versus sphere radius

\begin{tabular}{|c|c|c|c|c|c|c|}
\hline Radius $(\mathrm{m})$ & $\mathrm{T}_{\min }(\mathrm{s})$ & $\mathrm{T}_{\max }(\mathrm{s})$ & $\Delta \mathrm{T}(\mathrm{s})$ & $\mathrm{T}_{\mathrm{m}}(\mathrm{s})$ & $\mathrm{P}(\mathrm{kW})$ & $\mathrm{T}_{\mathrm{P}}(\mathrm{s})$ \\
\hline 1.5 & 1.45 & 3.30 & 1.85 & 2.38 & 7 & 2.57 \\
\hline 2.5 & 2.30 & 3.60 & 1.30 & 2.95 & 10 & 2.90 \\
\hline 4.0 & 3.20 & 4.10 & 0.90 & 3.65 & 14 & 3.67 \\
\hline
\end{tabular}

Where:

- $T_{\min }$ (s) is the minimum wave period value where the average power that can be transferred to the electronic converter is positive.

- $T_{\max }(\mathrm{s})$ is the maximum wave period value where the average power that can be transferred to the electronic converter is positive.

- $\Delta T(\mathrm{~s})$ is the work period bandwidth included between $T_{\min }$ and $T_{\max }$.

- $T_{m}(\mathrm{~s})$ is the medium value of the work period bandwidth.

- $\quad P(\mathrm{~kW})$ represents the maximum power that can be delivered to the first converter when reactive control is applied.

- $T_{p}$ (s) is the wave period value where this maximum power occurs.

Period values where the extracted power is positive are only considered, in order to limit the study to the area where the generator power can be extracted.

When sphere radius increases, the work period bandwidth decreases but the maximum power transferred to the electronic converter increases. Thus, when the device size increases, the period bandwidth where energy can be extracted from waves diminishes.

It can also be noted that when the radius increases, so do the bandwidth medium value $T_{m}$ and the natural frequency. As power exchanges are almost zero in resonance, the bandwidth moves to the right, towards bigger periods. Thus, the medium value of the work period bandwidth depends on the device size. When the sphere radius increases $60 \%, T_{m}$ (s) also increases about $24 \%$. On the other hand, when the sphere radius diminishes $40 \%, T_{m}(\mathrm{~s})$ diminishes around 19\%.

\section{Generator resistance sensitivity}

Table III shows different results when the generator resistance is modified for a $2.5 \mathrm{~m}$ radius sphere.

Table III. - Power and periods versus generator resistance

\begin{tabular}{|c|c|c|c|c|c|c|}
\hline $\begin{array}{c}\text { Resistance } \\
(\Omega)\end{array}$ & $\mathrm{T}_{\min }(\mathrm{s})$ & $\mathrm{T}_{\max }(\mathrm{s})$ & $\Delta \mathrm{T}(\mathrm{s})$ & $\mathrm{T}_{\mathrm{m}}(\mathrm{s})$ & $\mathrm{P}(\mathrm{kW})$ & $\mathrm{T}_{\mathrm{P}}(\mathrm{s})$ \\
\hline 0.20 & 2.10 & 4.10 & 2.00 & 3.10 & 10.90 & 3.30 \\
\hline 0.45 & 2.30 & 3.60 & 1.30 & 2.95 & 10 & 2.90 \\
\hline
\end{tabular}


When generator resistance increases, the bandwidth and the maximum power transferred to the electronic converter decrease. Thus, the electric power that can be injected into the grid diminishes when the losses increase. Table III shows that if the generator resistance increases above $50 \%$, the power transferred to the first converter diminishes only $2 \%$, whereas if a higher cable cross section is used, and thus resistance is reduced around $50 \%$, there could be injected almost $10 \%$ additional power into the grid. It can also be noted that when the resistance increases, the work bandwidth gets narrower. Table III shows that if the generator resistance increases above $50 \%$, the bandwidth diminishes around $20 \%$, whereas if the resistance is reduced around $50 \%$, the bandwidth increases in the same way (approximately $50 \%)$.

\section{Conclusions}

This paper shows the formulation that may be applied to calculate, not the quantity of energy catched from waves by a PMLG, but the one transferred from the linear generator to the power converter in regular waves taken into account the losses. It also has been highlighted how the oscillating system size and the generator resistance affect to the work bandwidth.

The results presented in this article reveal that the optimal reactive control strategy can be highly inefficient if the copper losses that occur in the linear generator are taken into account.

Future works will focus on modifying the reactive control strategy in order to increase the quantity and to improve the conditions in which electric power is delivered to the grid.

\section{Acknowledgements}

This work is part of the project ENE2010-18867, financed by the Spanish Ministry of Science and Education. The authors are also grateful for the financial support provided by Focus-Abengoa Foundation.

\section{References}

[1] M.A. Mueller, "Electrical generators for direct drive wave energy converters," IEE Proceedings, Generation, Transmission and Distribution, Vol. 149, no. 4, pp. 446 - 456, Jul. 2002.

[2] J. Falnes, "Ocean Waves and Oscillating Systems." Cambridge University Press, 2002.

[3] J.K.H. Shek, D.E. Macpherson, M.A. Mueller and J. Xiang "Reaction force control of a linear electrical generator for direct drive wave energy conversion", IET Renew. Power Gener. 1, (1), pp. 17-24, 2007.

[4] P.C. Krause, O. Wasynczuk and S.D. Sudhoff, "Analysis of Electric Machinery and Drive Systems." IEEE Press, 2002.

[5] A. García, D. Montoya, A. de la Villa, "Control of Hydrodynamic Parameters of Wave Energy Point Absorbers using Linear Generators and VSC-based Power Converters Connected to the Grid", In: 8th Intern. Conf. on Renewable Energies and Power Quality ICREPQ (March 2010).

[6] F.Wu; X. P. Zhang, P. Ju, M. J. H. Sterling, "Modelling and Control of AWS-Based Wave Energy Conversion System Integrated Into Power Grid", IEEE Trans. Power System (Aug. 2008). Vol. 23, no 3, pp. 1196-1204.

[7] F. Wu, X. Ping, P. Ju and H. Sterling, "Optimal Control for AWS-Based Wave Energy Conversion System", IEEE Trans. Power System (Nov. 2009). Vol. 24, no 3, pp. 1747-1755.

[8] A. Ferrero, G. Superti-Furga,"A New Approach to the Definition of Power Components in Three-Phase Systems under Non-sinusoidal Conditions", IEEE Trans. Instrumentation and Measurement (1991). Vol.40, n³, pp. 568-577.

[9] TH. Havelock, "Waves due to a floating sphere making periodic heaving oscillations", In: Proc. of the Royal Society (1955), Vol.231, n¹184, pp.1-7.

[10] O. Danielsson, M. Eriksson and M. Leijon,"Study of a longitudinal flux permanent magnet linear generator for wave energy converters", (2006), International Journal of Energy Research; Vol. 30, pp.1130-1145. 